\title{
A case of sustained ventricular tachycardia associated with severe ventricular dysfunction (tachycardiomyopathy)
}

\author{
K Sivakumar ${ }^{1}$, Priyanthi Molligoda ${ }^{2}$ \\ Sri Lanka Journal of Child Health, 2003; 32: 77-9
}

(Key words: tachycardiomyopathy, Belhassen ventricular tachycardia)

\section{Introduction}

Belhassen ventricular tachycardia (VT) is an idiopathic tachycardia occurring in structurally normal hearts. The mechanism of this tachycardia is a micro re-entrant focus in the left posterior fascicle of the left bundle, producing a broad QRS tachycardia of right bundle branch block (RBBB) morphology and left axis deviation (LAD). Sustained tachycardia, either of ventricular or supraventricular origin, lasting for hours to days with very fast rates, can result in dilatation of the ventricles and progressive reduction of their systolic contractility. This manifests clinically with cardiomegaly and echocardiographically as dilated cardiomyopathy. This clinical entity called tachycardiomyopathy differs from the usual types of dilated cardiomyopathy in being totally reversible on termination of the tachycardia. We describe a 4 year old child with sustained VT over 3 days resulting in severe ventricular dysfunction.

\section{Case report}

A 4 year old male child, who was previously well, had a history of cough, rapid breathing, and not doing well for three days prior to admission. The child was detected to have a fast heart rate $(230 / \mathrm{min})$ by the paediatrician and admitted to Durdans hospital, Colombo on $14^{\text {th }}$ November 2002. Echocardiographic evaluation in hospital showed dilatation and severely impaired contractility of both ventricles. He was diagnosed as having dilated cardiomyopathy and referred to Apollo hospital the next day, three days after the onset of his symptoms.

On clinical examination, he was normally grown for his age with a heart rate of $230 / \mathrm{min}$, low pulse volume and a blood pressure of $80 / 60 \mathrm{~mm} \mathrm{Hg}$. There was mild cardiomegaly. Echocardiogram (ECHO) showed significantly impaired ventricular function with an ejection fraction (EF) of $20 \%$. His electrocardiogram (EGG) (Figure 1) showed a ventricular rate of $214 / \mathrm{min}$, broad QRS tachycardia of RBBB morphology, QRS axis of $75^{\circ}$ and LAD. Careful analysis of $\mathrm{P}$ and QRS waves showed V-A dissociation; the QRS rate was 214/min and the P rate 102/min; P waves are shown in arrows (Figure 2). Based on the EGG features, a diagnosis of left posterior fascicular VT was made

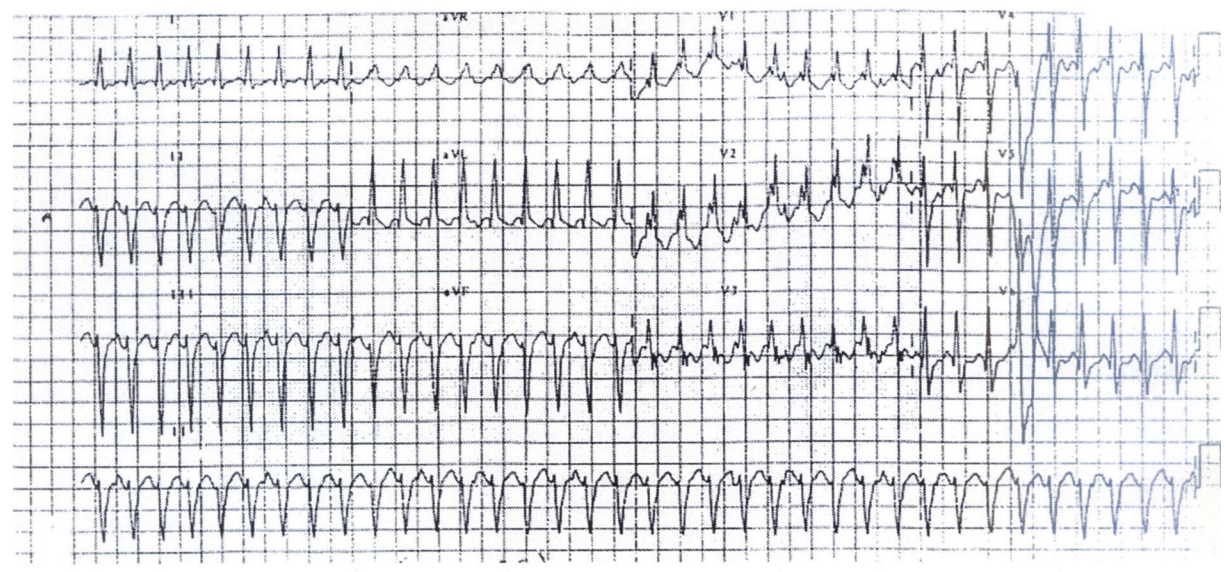

Figure 1

${ }^{1}$ Consutant Paediatric Cardiologist, ${ }^{2}$ Consultant Paediatrician, Apollo Hospitals, Colombo.

(Received on 25 Feb 2003) 


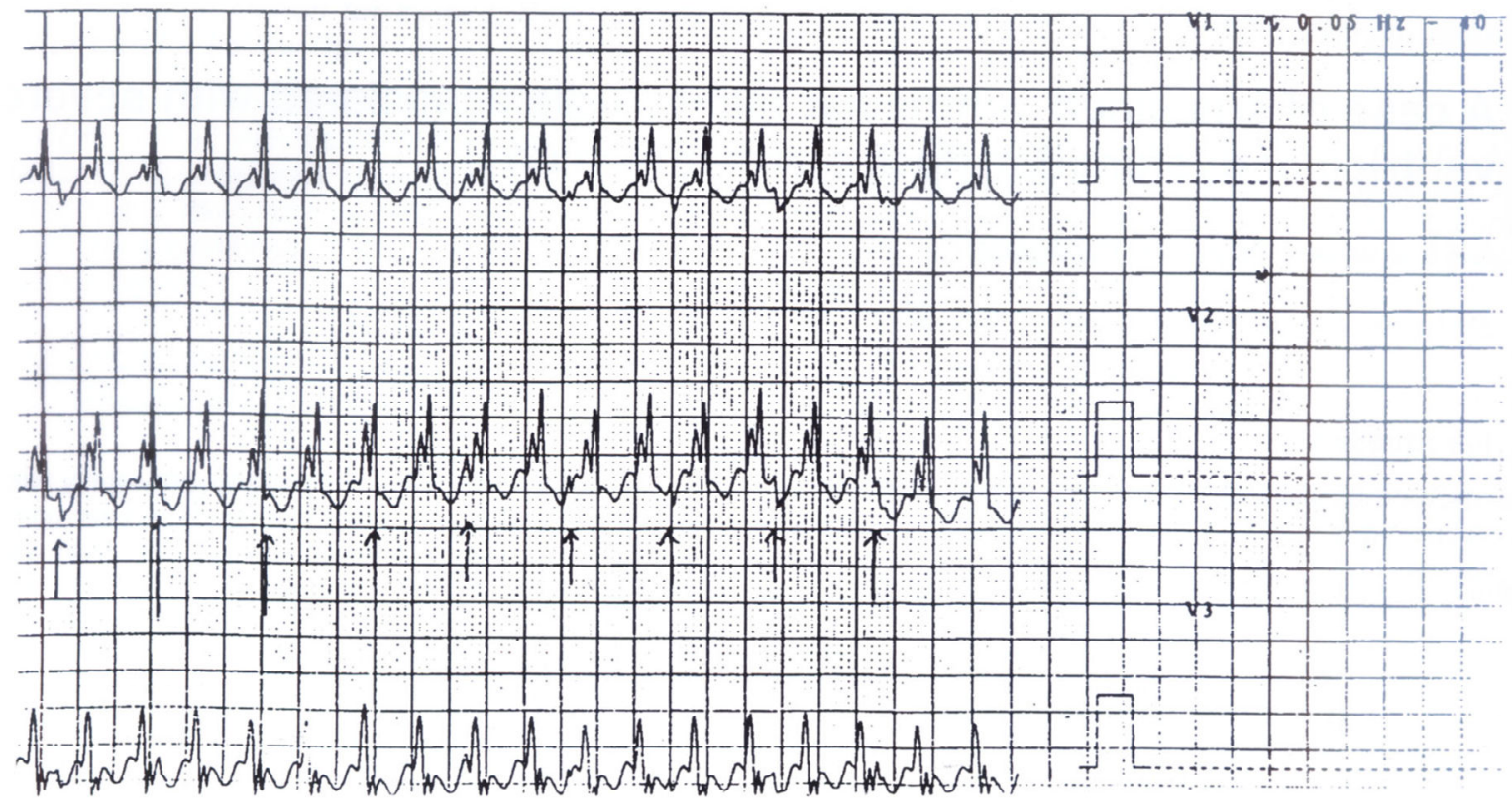

Figure 2

Initial trials to control the tachycardia with intravenous (IV) adenosine (150mg/kg rapid bolus) and metoprolol (two doses of $0.1 \mathrm{mg} / \mathrm{kg}$ ) failed. Even though this VT is well known to be verapamil sensitive, the ECHO finding of severe ventricular dysfunction precluded use of IV verapamil. The tachycardia was terminated using amiodarone $(5 \mathrm{mg} / \mathrm{kg}$ bolus over 30 minutes followed by infusion of $0.6 \mathrm{mg} / \mathrm{kg} /$ hour) within one hour. Subsequently the patient was started on oral verapamil $1 \mathrm{mg} / \mathrm{kg}$ once in 6 hours. After 72 hours of termination of the tachycardia, the ECHO showed total normalization of ventricular function and the EF improved to $55 \%$. There was no recurrence of tachycardia till the last follow-up.

\section{Discussion}

Sustained tachycardia with very fast rates, either of ventricular or supraventricular origin, lasting for hours to days, can result in dilatation of the ventricles and progressive reduction of their systolic contractility. This clinical entity, called tachcardiomyopathy, differs from the usual types of dilated cardiomyopathy in being totally reversible on termination of the tachycardia ${ }^{1}$. Identification of this entity is clinically important since termination of tachycardia needs usage of drugs like calcium channel blockers and betablockers which reduce inotropicity of the heart further. On the contrary, drugs to improve systolic contractility of failing ventricles, like catecholamine infusions which are administered in dilated cardiomyopathy, are contraindicated since they perpetuate tachycardia and further worsen systolic contractility of ventricles.

In any child presenting with tachyarrhythmia, a 12 lead EGG has to be recorded to differentiate supraventricular tachycardia (SVT) since the treatment strategies and prognoses differ. In this patient, EGG features of broad QRS tachycardia, superiorly directed QRS axis and V-A dissociation established the diagnosis of VT. Three additional features viz. RBBB morphology, LAD and absence of structural abnormality of the heart on ECHO narrowed the diagnosis to IDIOPATHIC LEFT POSTERIOR FASCICULAR VT (Belhassen VT).

The three common types of VT which occur in structurally normal heart (idiopathic VT) ${ }^{1}$ are (i) Left fascicular VT (ii) Left outflow tract VT and (iii) Right ventricular outflow tract VT. Fascicular VT are further classified as (i) Left posterior fascicular VT, the commonest form with RBBB morphology and leftward QRS axis, (ii) Left anterior fascicular VT with RBBB morphology and right axis and (iii) Upper septal fascicular VT, the rarest with normal QRS and normal axis ${ }^{1}$. The left posterior fascicular tachycardia (Belhassen VT), is also known as verapamil sensitive VT and originates from a micro reentrant focus near the posterior fascicle of the left bundle $^{2}$. This produces a broad QRS tachycardia of RBBB morphology and LAD, the QRS axis lying between $45^{\circ}$ and $90^{\circ}$. The tachyarrhythmia responds well to verapamil but may not respond to many other 
anti-arrhythmic drugs making recognition of this arrhythmia very crucial for management ${ }^{2}$.

Cardiomyopathy, defined as primary myocardial dysfunction, denotes a dismal prognosis short of heart transplantation ${ }^{3}$. Some causes of "treatable cardiomyopathy" include ventricular outflow tract obstructions, coronary anomalies, sustained tachyarrhythmia and certain metabolic abnormalities. Sustained SVT and VT result in impairment of ventricular function and this entity called tachycardiomyopathy has been recognized since the early $90 \mathrm{~s}^{4,5,6}$. The termination of tachycardia results in very early normalization of left ventricular function ${ }^{7,8,9}$. Failure to recognize this entity may lead to continuation of the tachycardia for long periods and leads to worsening of the ventricular function and sudden cardiac deaths ${ }^{10}$. Electrical and molecular abnormalities have been identified in the role of fast ventricular rates in development of tachycardiomyopathy and remodelling and dilatation of the heart ${ }^{11}$.

\section{Acknowledgments}

We thank Dr. Stella de Silva, Consultant Paediatrician, Durdan's Hospital, Colombo for giving us the patient and Mr Jayanth, Apollo Hospital for the technical support.

\section{References}

1. Nogami A. Idiopathic left ventricular tachycardia: assessment and treatment. Card Electrophysiol Rev 2002; 6: 448-57.

2. Winter K, Gossen N, Ulbricht L J, Liersch R. Verapamil sensitive ventricular tachycardia with myocardial failure in a 2 year old child. $Z$ Kardiol 1999; 88: 369-73.

3. Prabhu S S, Dalvi B V. Treatable cardiomyopathies - A review. Indian J Pediatr 2000; 67: 279-82.
4. Brugada P. Changas disease and tachycardiomyopathy. Arq Bras Cardiol 1991; 56: 5-7.

5. Fenelon G, Wijns W, Andries E, Brugada P. Tachycardiomyopathy: mechanisms and clinical implications. Pacing Clin Electrophysiol 1996 ; 19: $95-106$.

6. Brugada P, Andries E. Tachycardiomyopathy: The most frequently unrecognised cause of heart failure? Acta Cardiol 1993; 48:165-9.

7. Juneja R, Shah S, Naik N, Kothari S S, Saxena A. Talwar K K. Management of cardiomyopathy resulting from incessant supraventricular tachycardia in infants and children. Indian Heart $J$ 2002; 54:176-80.

8. Noe P, Van Driel V, Wittkampf F, Sreeram N. Rapid recovery of cardiac function after catheter ablation of persistent junctional reciprocating tachycardia in children. Pacing Clin Electrophysiol 2002; 25:191-4.

9. Sternick E B, Bahia F C, Gontijo Filho B, Vrandecic M O. Cardiomyopathy induced by incessant ventricular tachycardia (tachycardiomyopathy) cure after control of arrhythmia. Arq Bras Cardiol 1992; 58:209-14.

10. Afonso M R, Franca H H. Sudden death and tachycardiomyopathy in a young man with incessant tachycardia. Arq Bras Cardiol 1992; 58: 303-6.

11. Schoonderwoerd B A, Van Gelder 1 C, van Veldhuisen D J, Tieleman R G, Grandjean J Q Bel $\mathrm{K} \mathrm{J}$ et al. Electrical remodelling and atrial dilatation during atrial tachycardia are influenced by ventricular rate role of developing tachycardiomyopathy. $J \quad$ Cardiovasc Electrophysiol 2001; 12:1404-10. 